\title{
Facile Fabrication of Stretchable Electrodes by Sedimentation of Ag Nanoparticles in PDMS Matrix
}

\author{
Jae-Yeop Kim ${ }^{1}$ and Kwang-Suk Jang $\mathbb{D}^{1,2}$ \\ ${ }^{1}$ Department of Applied Chemistry, Hanyang University, Ansan, Gyeonggi-do 15588, Republic of Korea \\ ${ }^{2}$ Department of Chemical and Molecular Engineering, Hanyang University, Ansan, Gyeonggi-do 15588, Republic of Korea \\ Correspondence should be addressed to Kwang-Suk Jang; kjang@hanyang.ac.kr
}

Received 11 November 2017; Revised 22 February 2018; Accepted 7 March 2018; Published 8 April 2018

Academic Editor: Raul Arenal

Copyright (c) 2018 Jae-Yeop Kim and Kwang-Suk Jang. This is an open access article distributed under the Creative Commons Attribution License, which permits unrestricted use, distribution, and reproduction in any medium, provided the original work is properly cited.

\begin{abstract}
This study reports a facile fabrication method for highly conductive stretchable electrodes composed of a conductive 0D nanomaterial filler and an elastomer matrix. The local volume fraction of the Ag nanoparticles at the composite surface could be significantly increased by the sedimentation of Ag nanoparticles in uncured polydimethylsiloxane (PDMS) fluid. The stretchable electrodes had a surface resistance of $0.910 \pm 0.509 \Omega /$ sq and were stretchable up to $100 \%$ of strain without the reduction of the electrical conductivity, demonstrating their potential as high-performance electrodes for applications in stretchable electronics.
\end{abstract}

\section{Introduction}

Stretchable electrodes are one of the key components of future electronic devices such as flexible and stretchable displays, strain sensors, actuators, nanogenerators, and wearable electronic devices [1-18]. Thus, simple and cost-effective processes for the fabrication of stretchable electrodes will be in high demand. Making composites with conductive materials and elastomers is the simplest route to obtaining stretchable electrodes [9-18]. Electrical conductivity and stretchability of the composites are affected significantly by volume fraction, morphology, and dispersion of the conductive materials [19-21]. It is well known that as the volume fraction of the conductive filler increases, the electrical conductivity of the composites increases and stretchability decreases. As conductive fillers, $1 \mathrm{D}$ and $2 \mathrm{D}$ nanomaterials such as metal nanowires, carbon nanotubes, and graphene have been widely used. These $1 \mathrm{D}$ or $2 \mathrm{D}$ nanomaterials can make junctions effectively in a nonconductive elastomer matrix because of their morphologies. Because the polymer matrix is not electrically conductive, the junction between conductive fillers is the electrical conduction pathway of the composites. Thus, the density of the junctions is strongly related to the electrical conductivity of the composites.
The $0 \mathrm{D}$ nanomaterials such as metal nanoparticles have rarely been used as conductive fillers for conductive composites. Because the percolation threshold of $0 \mathrm{D}$ nanomaterials is high, a very high volume fractions of the conductive filler is required to achieve high conductivity in the composites and, as a result, stretchability could be significantly reduced. Rosset et al. reported asymmetric metal nanoparticle/elastomer stretchable electrodes, where metal nanoparticles were implanted onto the surface of the elastomer substrate [22]. By the plasma-based ion implantation method, compact aggregations of metal nanoparticle shells on the elastomer substrates could be obtained. The surface resistance and stretchability were controlled by the ion dose. The minimum surface resistance was $\sim 100 \Omega / \mathrm{sq}$, and the maximum strain was $175 \%$. Because of the asymmetric structure, conductive and stretchable composites with OD nanomaterials could be achieved. However, the fabrication process for the asymmetric stretchable electrodes is neither simple nor costeffective. More importantly, the surface resistance needs to be decreased for a wide range of applications.

In this paper, we report a simple and cost-effective process for the fabrication of asymmetric, stretchable, and highly conductive metal nanoparticle/elastomer composites. Agglomerations of $\mathrm{Ag}$ nanoparticles were formed at the 


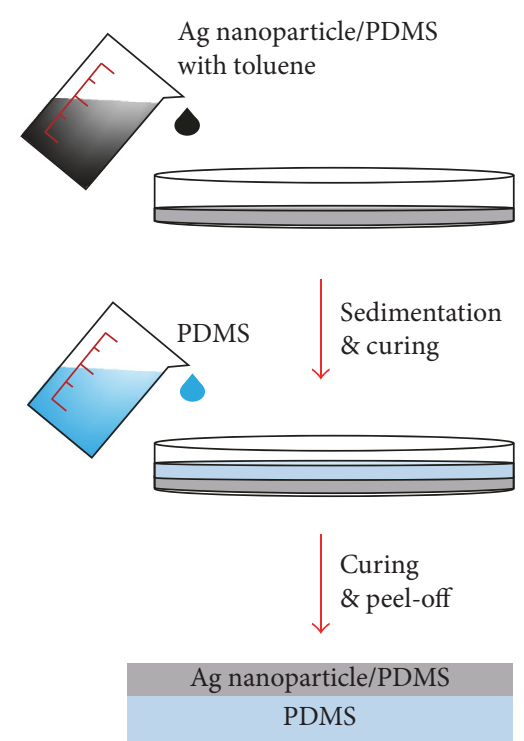

Scheme 1: Fabrication procedure for the asymmetric stretchable electrode.

surface of the composite films by the sedimentation of Ag nanoparticles in uncured polydimethylsiloxane (PDMS) fluid. These agglomerations are highly conductive with a minimum surface resistance of $0.431 \Omega /$ sq. The asymmetric Ag nanoparticle/PDMS composite films are stretchable up to $100 \%$ of strain. In general, as the strain of the composites with conductive fillers increases, the electrical conductivity decreases. However, interestingly, the surface resistance of the asymmetric Ag nanoparticle/PDMS composite films decreases with increased tensile strain. This may be due to the electrical conduction pathways created by out-of-plane compaction of the Ag nanoparticles caused by the in-plane strain.

\section{Experimental}

Ag nanoparticles (diameter of $<100 \mathrm{~nm}$ ) were purchased from Sigma-Aldrich. The PDMS base polymer and curing agent (Sylgard 184) were purchased from Dow Corning. Toluene (99.8\%) was purchased from Samchun Pure Chemical. All materials were used as received. The fabrication procedure of the asymmetric stretchable electrode is shown in Scheme 1. Ag nanoparticles (0.45 g), the PDMS base polymer $(0.285 \mathrm{~g})$, and curing agent $(0.015 \mathrm{~g})$ were mixed using a mortar and pestle for $10 \mathrm{~min}$. Then, $2.5 \mathrm{~g}$ of toluene was added and the mixture was mixed with a mortar and pestle for $10 \mathrm{~min}$. The $3 \mathrm{~g}$ mixture was poured into an $\mathrm{Al}$ foil cup with a diameter of $2 \mathrm{~cm}$. For the sedimentation of the Ag nanoparticles, the mixture was aged at room temperature for $1 \mathrm{hr}$. Then, the mixture in the $\mathrm{Al}$ foil cup was annealed at $100^{\circ} \mathrm{C}$ on a hot plate for $2 \mathrm{~h}$ to remove residual toluene. For fabrication of the bilayer-structured film, $1.14 \mathrm{~g}$ of the PDMS base polymer and $0.006 \mathrm{~g}$ of curing agent were mixed using a mortar and pestle for $10 \mathrm{~min}$. The $1.1 \mathrm{~g}$ of uncured PDMS was poured onto the Ag nanoparticle/PDMS composite layer and annealed at $150^{\circ} \mathrm{C}$ on a hot plate for more than $20 \mathrm{~h}$. The asymmetric

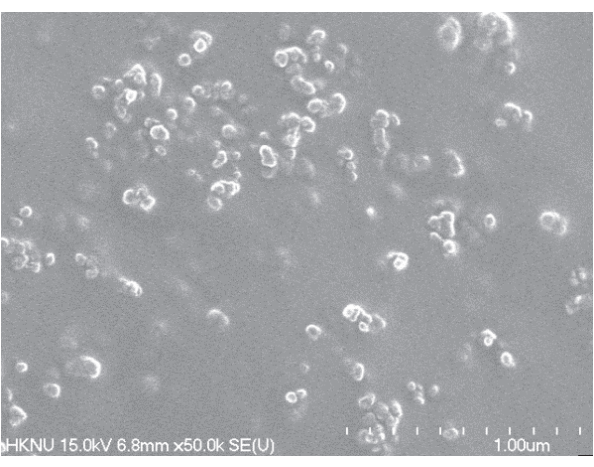

FIGURE 1: SEM image of the Ag nanoparticles.

stretchable electrode was peeled off and cut into a rectangular shape with dimensions of $12 \mathrm{~mm} \times 17 \mathrm{~mm}$. Thicknesses of the Ag nanoparticle/PDMS composite layer and PDMS layer were $\sim 0.2$ and $\sim 1.5 \mathrm{~mm}$, respectively. For the dispersion stability test, $30 \mathrm{mg}$ of $\mathrm{Ag}$ nanoparticles was dispersed in the uncured PDMS $(2.8 \mathrm{~g})$ and the mixture of uncured PDMS (0.3 g) and toluene (2.5 g), respectively, using a mortar and pestle. The sheet resistances of unstreched films were measured using the standard four-point probe method with a CMT-SR1000N (AIT, Republic of Korea). The resistances of stretched films were extracted from the slope of the straightline fit of the $V-I$ curve measured with a Keithley 2200 power source, a Keithley 2460 source meter, and a Keithley 2182A nanovoltmeter. Two edges of samples were gripped by electrodes with a width of $20 \mathrm{~mm}$, which were separated by a distance of $10 \mathrm{~mm}$.

\section{Results and Discussion}

For fabrication of the conductive composite layer, we used PDMS as a stretchable matrix and Ag nanoparticles as a conductive filler. To the Ag nanoparticle/PDMS composite layer, an additional PDMS layer was introduced as a substrate, as shown in Scheme 1. The average diameter of the Ag nanoparticles was $65 \pm 21 \mathrm{~nm}$ (Figure 1). The Ag nanoparticles up to $60 \mathrm{wt} \%$ were well dispersed in uncured PDMS liquid by mixing a pestle and mortar. At first, we prepared Ag nanoparticle/PDMS composite films without adding toluene in the mixture; the mass fraction of $\mathrm{Ag}$ nanoparticles was varied from 10 to $60 \mathrm{wt} \%$. However, the Ag nanoparticle/PDMS composite films prepared without toluene were not electrically conductive. The percolation threshold of the Ag nanoparticles in the PDMS matrix might be higher than $60 \mathrm{wt} \%$. The percolation threshold of $0 \mathrm{D}$ nanomaterials could be predicted with their diameter using a model by Kim et al. [19-21]. The calculated percolation threshold of the Ag nanoparticles with a diameter of $65 \mathrm{~nm}$ in PDMS is $34 \mathrm{vol} \%$, which is equivalent to $>80 \mathrm{wt} \% \mathrm{Ag}$ nanoparticles. The $0 \mathrm{D}$ nanomaterials such as metal nanoparticles have been rarely used as conductive fillers because of their high percolation threshold.

In this study, we propose an asymmetrically structured Ag nanoparticles/PDMS composite film for applications in 

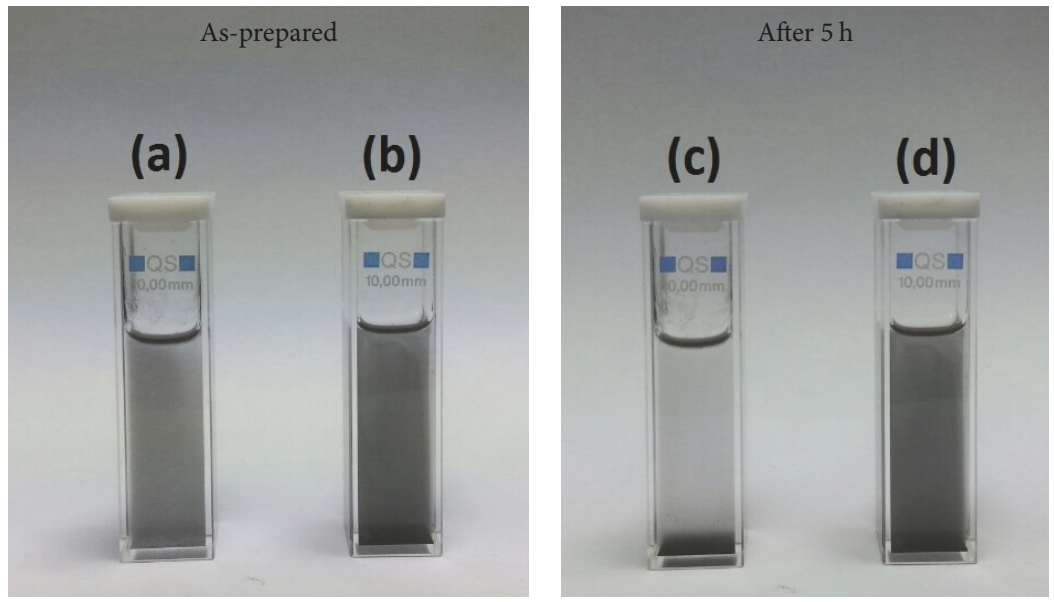

FIGURE 2: Photographs of the as-prepared and $5 \mathrm{hr}$ aged Ag nanoparticle/uncured PDMS mixtures prepared (a, c) with and (b, d) without toluene.

stretchable electrodes. We used toluene as an additive for the asymmetric distribution of $\mathrm{Ag}$ nanoparticles within the PDMS matrix. In the liquid mixture of uncured PDMS and toluene, the dispersion stability of the Ag nanoparticles is poor, and sedimentation of the $\mathrm{Ag}$ nanoparticles occurs. The Ag nanoparticles were dispersed in the uncured PDMS with and without toluene to compare the stabilities of the dispersions. As shown in Figure 2, the Ag nanoparticles were well dispersed in the mixtures with and without toluene. The Ag nanoparticle dispersions exhibit black colour because the $\mathrm{Ag}$ nanoparticles have a broad size distribution from a few nanometers to $100 \mathrm{~nm}$ and there might be secondary agglomeration. After $5 \mathrm{~h}$, only the Ag nanoparticles in the uncured PDMS with toluene had precipitated. Because the Ag nanoparticles used in this study are not coated by hydrophobic ligands, the Ag nanoparticles are not well dispersed in hydrophobic solvents such as toluene. Toluene might be a bad solvent for dispersion of the Ag nanoparticles. Because of the addition of toluene, sedimentation of the Ag nanoparticles in the mixture could occur, forming the asymmetric structure and, in so doing, increasing the local volume fraction of the Ag nanoparticles at the surface of the composite films. The presence of toluene induces the sedimentation of the Ag nanoparticles in the mixture. Thus, evaporation speed of toluene should affect the sedimentation. For the sufficient sedimentation of the Ag nanoparticles, the mixture, poured into an $\mathrm{Al}$ foil cup, was aged at room temperature for $1 \mathrm{hr}$ before annealing at $100^{\circ} \mathrm{C}$ to remove residual toluene. We prepared asymmetric Ag nanoparticle/PDMS composite films where the mass fraction of $\mathrm{Ag}$ nanoparticles was varied from 10 to $60 \mathrm{wt} \%$. Figure 3 and Table 1 show the surface resistances of the asymmetric composite films. The asymmetric $\mathrm{Ag}$ nanoparticle/PDMS composite films with 10 and $20 \mathrm{wt} \% \mathrm{Ag}$ nanoparticles were not electrically conductive. The surface resistance of the asymmetric composite films with $30 \mathrm{wt} \% \mathrm{Ag}$ nanoparticles was $1760 \pm 1150 \Omega /$ sq. As the relative content of Ag nanoparticles increases, the surface resistance decreases. The surface resistance of the asymmetric composite films with $60 \mathrm{wt} \% \mathrm{Ag}$ nanoparticles was $0.910 \pm 0.509 \Omega / \mathrm{sq}$. The
TABLE 1: The Ag nanoparticle composition-dependent surface resistances of the asymmetric stretchable electrodes.

\begin{tabular}{lc}
\hline wt $\%$ Ag nanoparticles & Surface resistance $[\Omega / s q]$ \\
\hline 10 & Not measurable \\
20 & Not measurable \\
30 & $1760 \pm 1150$ \\
40 & $1150 \pm 772$ \\
50 & $21.6 \pm 17.1$ \\
60 & $0.910 \pm 0.509$ \\
\hline
\end{tabular}

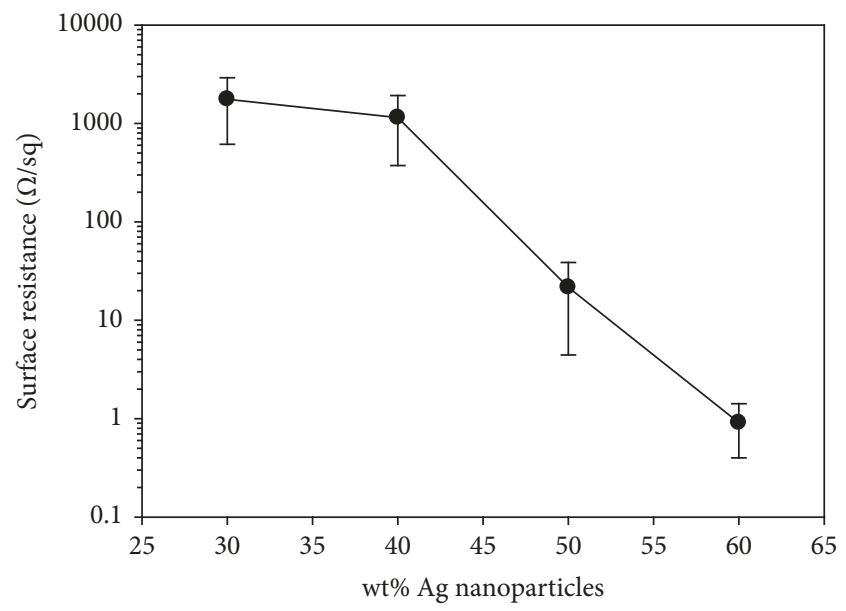

FIGURE 3: The Ag nanoparticle composition-dependent surface resistances of the asymmetric stretchable electrodes.

minimum surface resistance was measured to be $0.431 \Omega / s q$. Using a simple sedimentation process, highly conductive composite films could be obtained using 0D nanomaterials as a conductive filler.

Figure 4(a) shows the scanning electron microscope (SEM) images of the cross-sectioned asymmetric $\mathrm{Ag}$ nanoparticle/PDMS composite film with $60 \mathrm{wt} \% \mathrm{Ag}$ 


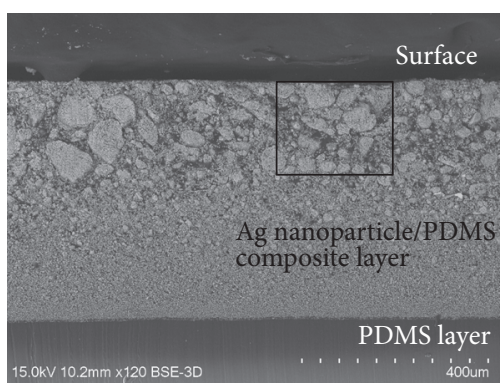

(a)

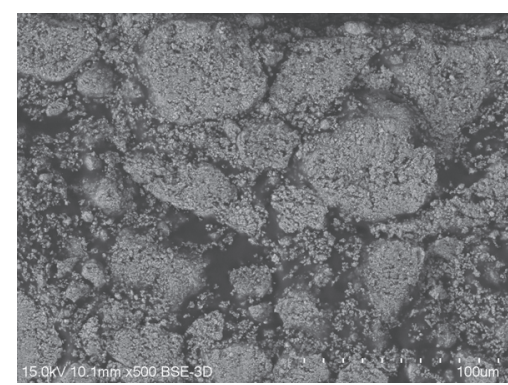

(b)

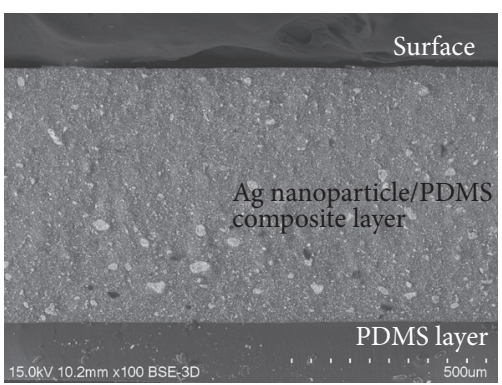

(c)

FIGURE 4: SEM images of the cross-sectioned Ag nanoparticle/PDMS composite films with $60 \mathrm{wt} \%$ Ag nanoparticles prepared (a, b) with and (c) without toluene. The box in (a) represents the area shown in (b).

nanoparticles. The box in Figure 4(a) represents the area shown in Figure 4(b). There are many secondary aggregates of the Ag nanoparticles with diameters in the range from 1 to $100 \mu \mathrm{m}$ at the surface. On the opposite side, the Ag nanoparticles are better dispersed and do not form secondary aggregates. The asymmetric structure of the composite film and its very high volume fraction of Ag nanoparticles on the surface are confirmed by SEM analysis. For comparison, the SEM image of the crosssectioned Ag nanoparticle/PDMS composite film with $60 \mathrm{wt} \% \mathrm{Ag}$ nanoparticles prepared without toluene is shown in Figure 4(c). Secondary aggregates of Ag nanoparticles with diameters in the range of 1 to $50 \mu \mathrm{m}$ are also observed in Figure $4(\mathrm{c})$. However, the density of the secondary aggregates is minimal, and they are randomly distributed. At the surface of the composite film prepared without toluene, the Ag nanoparticles are well dispersed and their volume fraction might be less than the percolation threshold. By addition of toluene to the Ag nanoparticle/uncured PDMS mixture, the dispersion stability of the Ag nanoparticles was decreased and their sedimentation could occur. The formation of secondary aggregates of Ag nanoparticles on the surface by sedimentation might be the origin of the extremely low surface resistances of the asymmetric Ag nanoparticle/PDMS composite films.

To investigate the potential of bilayers composed of the highly conductive asymmetric Ag nanoparticle/PDMS composite layer with $60 \mathrm{wt} \%$ Ag nanoparticles and a PDMS substrate layer as a stretchable electrode, the strain-dependent resistances were measured (Figure 5). The prepared asymmetric stretchable electrodes were stretchable up to $100 \%$ of strain without plastic deformation or cracking. In most conductive filler/elastic matrix composites, as the strain increases, the resistance increases because of the increased average interfiller distance. However, interestingly, the resistance of the asymmetric stretchable electrodes decreased with the tensile strain below 100\% (Figure 5). At 20\% strain, the stretchable electrodes showed the least resistance. As the strain increases above $20 \%$, the resistance increases. The resistance at the strain of $100 \%$ is still less than that of the unstrained state. By the simple sedimentation process, electrodes that are stretchable up to $100 \%$ of strain without the reduction of the electrical conductivity could

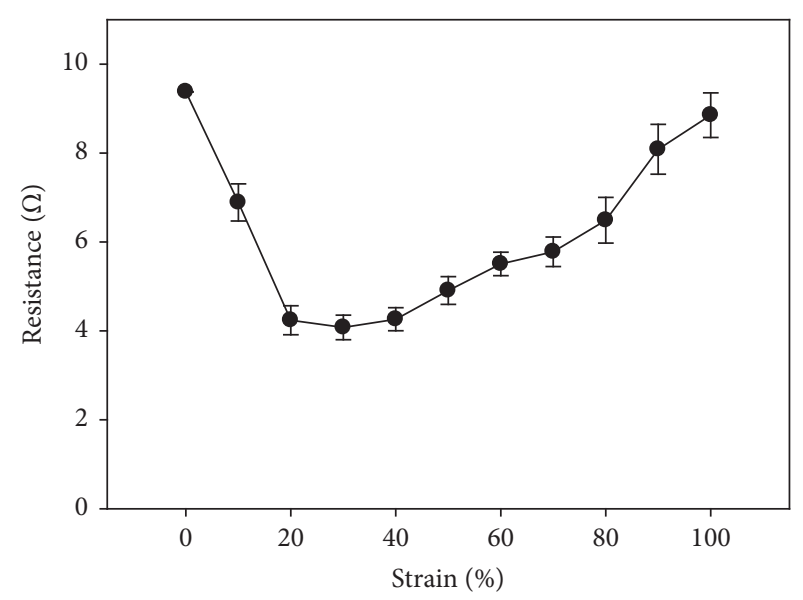

Figure 5: The strain-dependent resistances of the asymmetric stretchable electrodes with the $60 \mathrm{wt} \% \mathrm{Ag}$ nanoparticle composite layer.

be fabricated. The electrical conductance of the conductive filler/elastic matrix composites is affected significantly by the average interfiller distance [19-21]. The decrease in resistance might be due to the decreased average distance between Ag nanoparticles caused by the tensile strain. In the unstrained electrode, electrical conduction pathways will be formed through the secondary aggregates of the Ag nanoparticles (Scheme 2(a)). In the stretched electrode, the electrical conduction pathways might be created well by the out-ofplane compaction of the Ag nanoparticles by the in-plane strain (Scheme 2(b)). The exceptionally high volume fraction of Ag nanoparticles at the surface might be the origin of the interesting strain-dependent resistance behavior.

\section{Conclusions}

In summary, we report a facile fabrication method for highly conductive stretchable electrodes with conductive 0D nanomaterials. Addition of toluene to the Ag nanoparticle/uncured PDMS mixture decreases the dispersion stability of the Ag nanoparticles and induces their sedimentation at 


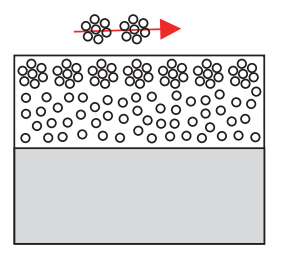

(a)

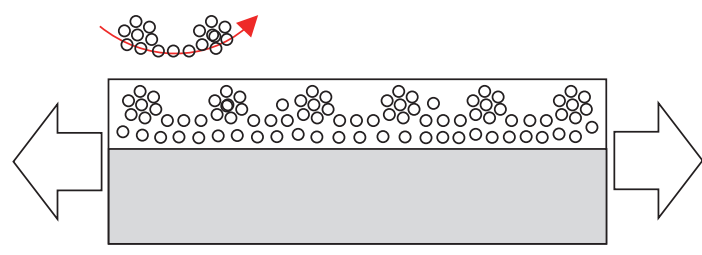

(b)

Scheme 2: Schematic diagrams of electrical conduction pathways of the (a) unstretched and (b) stretched asymmetric stretchable electrodes.

the surface. After curing the PDMS matrix, a very high volume fraction of Ag nanoparticles could be obtained at the surface of the composite film. The minimum surface resistance of the asymmetric Ag nanoparticle/PDMS composite film was measured to be $0.431 \Omega /$ sq. The stretchable electrodes with the asymmetric Ag nanoparticle/PDMS composite film with the $60 \mathrm{wt} \% \mathrm{Ag}$ nanoparticles were stretchable up to $100 \%$ of strain without plastic deformation, cracking, or reduction of the electrical conductivity. We anticipate that this facile sedimentation process will be applicable to the fabrication of various inorganic filler/elastomer composites for stretchable electronic and energy device applications.

\section{Conflicts of Interest}

The authors declare that there are no conflicts of interest regarding the publication of this paper.

\section{Acknowledgments}

This work was supported by the National Research Foundation of Korea (NRF) grant funded by the Korean Government (Ministry of Education) (no. 2016R1D1A1B03930409).

\section{References}

[1] J. A. Rogers, T. Someya, and Y. Huang, "Materials and mechanics for stretchable electronics," Science, vol. 327, no. 5973, pp. 1603-1607, 2010.

[2] H. C. Ko, M. P. Stoykovich, J. Song et al., "A hemispherical electronic eye camera based on compressible silicon optoelectronics," Nature, vol. 454, no. 7205, pp. 748-753, 2008.

[3] T. Sekitani, H. Nakajima, H. Maeda et al., "Stretchable activematrix organic light-emitting diode display using printable elastic conductors," Nature Materials, vol. 8, no. 6, pp. 494-499, 2009.

[4] C. Yan, W. Kang, J. Wang et al., "Stretchable and wearable electrochromic devices," ACS Nano, vol. 8, no. 1, pp. 316-322, 2014.

[5] K. Xie and B. Wei, "Materials and structures for stretchable energy storage and conversion devices," Advanced Materials, vol. 26, no. 22, pp. 3592-3617, 2014.

[6] M. L. Hammock, A. Chortos, B. C.-K. Tee, J. B.-H. Tok, and Z. Bao, "25th Anniversary article: the evolution of electronic skin (E-Skin): a brief history, design considerations, and recent progress," Advanced Materials, vol. 25, no. 42, pp. 5997-6038, 2013.
[7] S. Yao and Y. Zhu, "Nanomaterial-enabled stretchable conductors: Strategies, materials and devices," Advanced Materials, vol. 27, no. 9, pp. 1480-1511, 2015.

[8] T. Cheng, Y. Zhang, W.-Y. Lai, and W. Huang, "Stretchable thinfilm electrodes for flexible electronics with high deformability and stretchability," Advanced Materials, vol. 27, no. 22, pp. 33493376, 2015.

[9] H.-C. Jung, J.-H. Moon, D.-H. Baek et al., "CNT/PDMS composite flexible dry electrodesfor long-term ECG monitoring," IEEE Transactions on Biomedical Engineering, vol. 59, no. 5, pp. 1472-1479, 2012.

[10] A. Mohammed and M. Pecht, "A stretchable and screenprintable conductive ink for stretchable electronics," Applied Physics Letters, vol. 109, no. 18, Article ID 184101, 2016.

[11] J. Y. Oh, G. H. Jun, S. Jin, H. J. Ryu, and S. H. Hong, "Enhanced Electrical Networks of Stretchable Conductors with Small Fraction of Carbon Nanotube/Graphene Hybrid Fillers," ACS Applied Materials \& Interfaces, vol. 8, no. 5, pp. 3319-3325, 2016.

[12] S. Chen, Y. Wei, X. Yuan, Y. Lin, and L. Liu, "A highly stretchable strain sensor based on a graphene/silver nanoparticle synergic conductive network and a sandwich structure," Journal of Materials Chemistry C, vol. 4, no. 19, pp. 4304-4311, 2016.

[13] Y. Lin, S. Liu, S. Chen, Y. Wei, X. Dong, and L. Liu, "A highly stretchable and sensitive strain sensor based on grapheneelastomer composites with a novel double-interconnected network," Journal of Materials Chemistry C, vol. 4, no. 26, pp. 63456352, 2016.

[14] H. Liu, Y. Li, K. Dai et al., "Electrically conductive thermoplastic elastomer nanocomposites at ultralow graphene loading levels for strain sensor applications," Journal of Materials Chemistry C, vol. 4, no. 1, pp. 157-166, 2015.

[15] S. Wang, X. Zhang, X. Wu, and C. Lu, “Tailoring percolating conductive networks of natural rubber composites for flexible strain sensors via a cellulose nanocrystal templated assembly," Soft Matter, vol. 12, no. 3, pp. 845-852, 2016.

[16] H. Liu, J. Gao, W. Huang et al., "Electrically conductive strain sensing polyurethane nanocomposites with synergistic carbon nanotubes and graphene bifillers," Nanoscale, vol. 8, no. 26, pp. 12977-12989, 2016.

[17] M. A. Darabi, A. Khosrozadeh, Q. Wang, and M. Xing, "Gum Sensor: A Stretchable, Wearable, and Foldable Sensor Based on Carbon Nanotube/Chewing Gum Membrane," ACS Applied Materials \& Interfaces, vol. 7, no. 47, pp. 26195-26205, 2015.

[18] J. Li, S. Zhao, X. Zeng et al., "Highly Stretchable and Sensitive Strain Sensor Based on Facilely Prepared Three-Dimensional Graphene Foam Composite," ACS Applied Materials \& Interfaces, vol. 8, no. 29, pp. 18954-18961, 2016.

[19] J. Li and J.-K. Kim, "Percolation threshold of conducting polymer composites containing 3D randomly distributed graphite 
nanoplatelets," Composites Science and Technology, vol. 67, no. 10, pp. 2114-2120, 2007.

[20] J. Li, P. C. Ma, W. S. Chow, C. K. To, B. Z. Tang, and J.-K. Kim, "Correlations between percolation threshold, dispersion state, and aspect ratio of carbon nanotubes," Advanced Functional Materials, vol. 17, no. 16, pp. 3207-3215, 2007.

[21] M. Park, J. Park, and U. Jeong, "Design of conductive composite elastomers for stretchable electronics," Nano Today, vol. 9, no. 2, pp. 244-260, 2014.

[22] S. Rosset, M. Niklaus, P. Dubois, and H. R. Shea, "Metal ion implantation for the fabrication of stretchable electrodes on elastomers," Advanced Functional Materials, vol. 19, no. 3, pp. 470-478, 2009. 


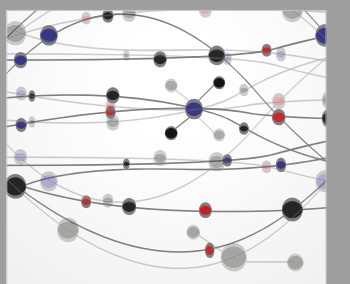

The Scientific World Journal
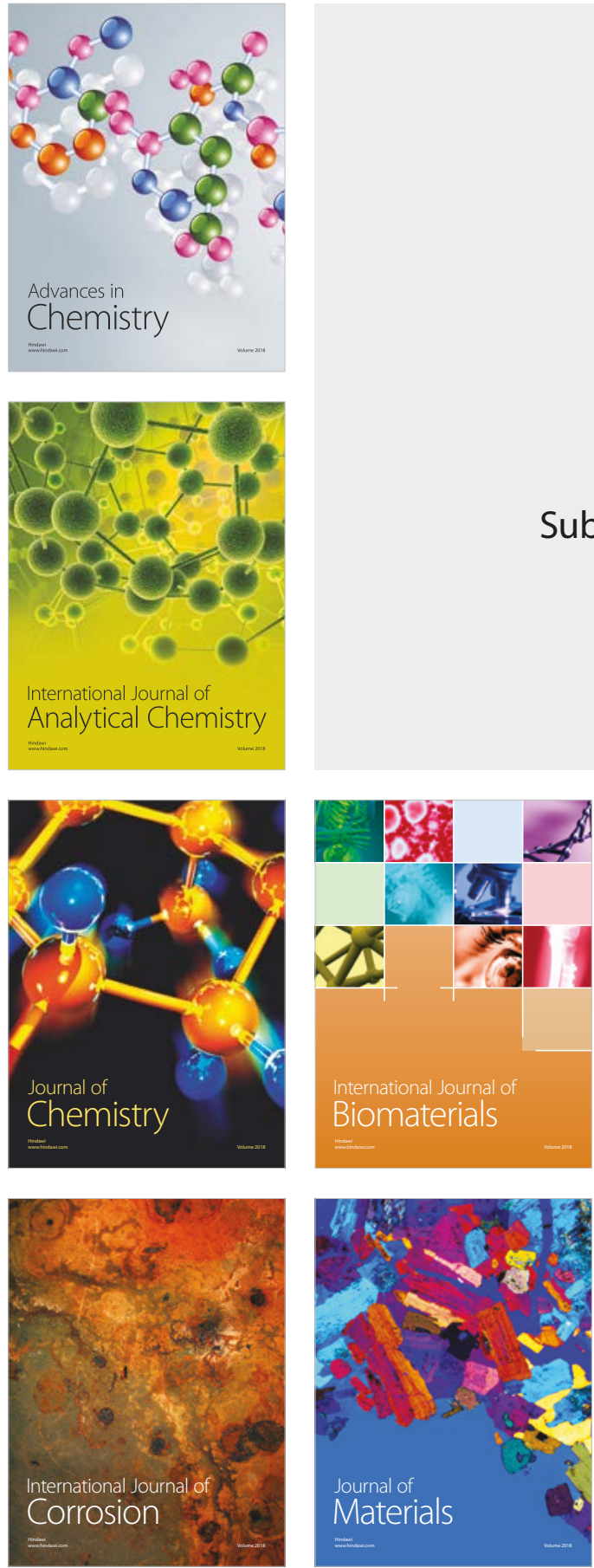

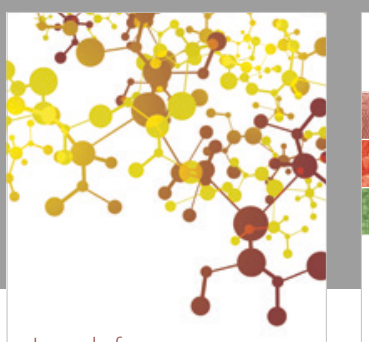

Journal of

Applied Chemistry
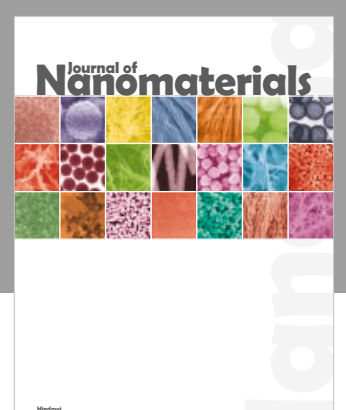

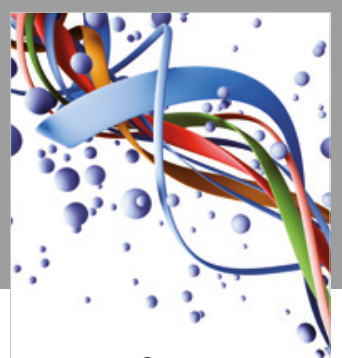

Scientifica

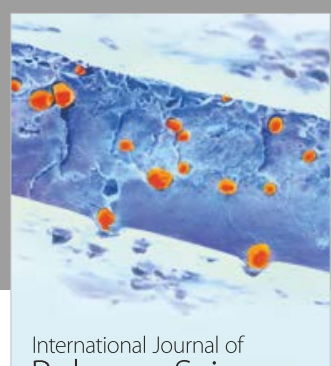

Polymer Science

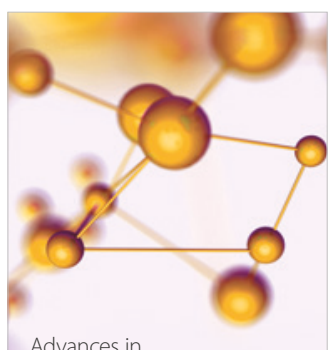

Physical Chemistry
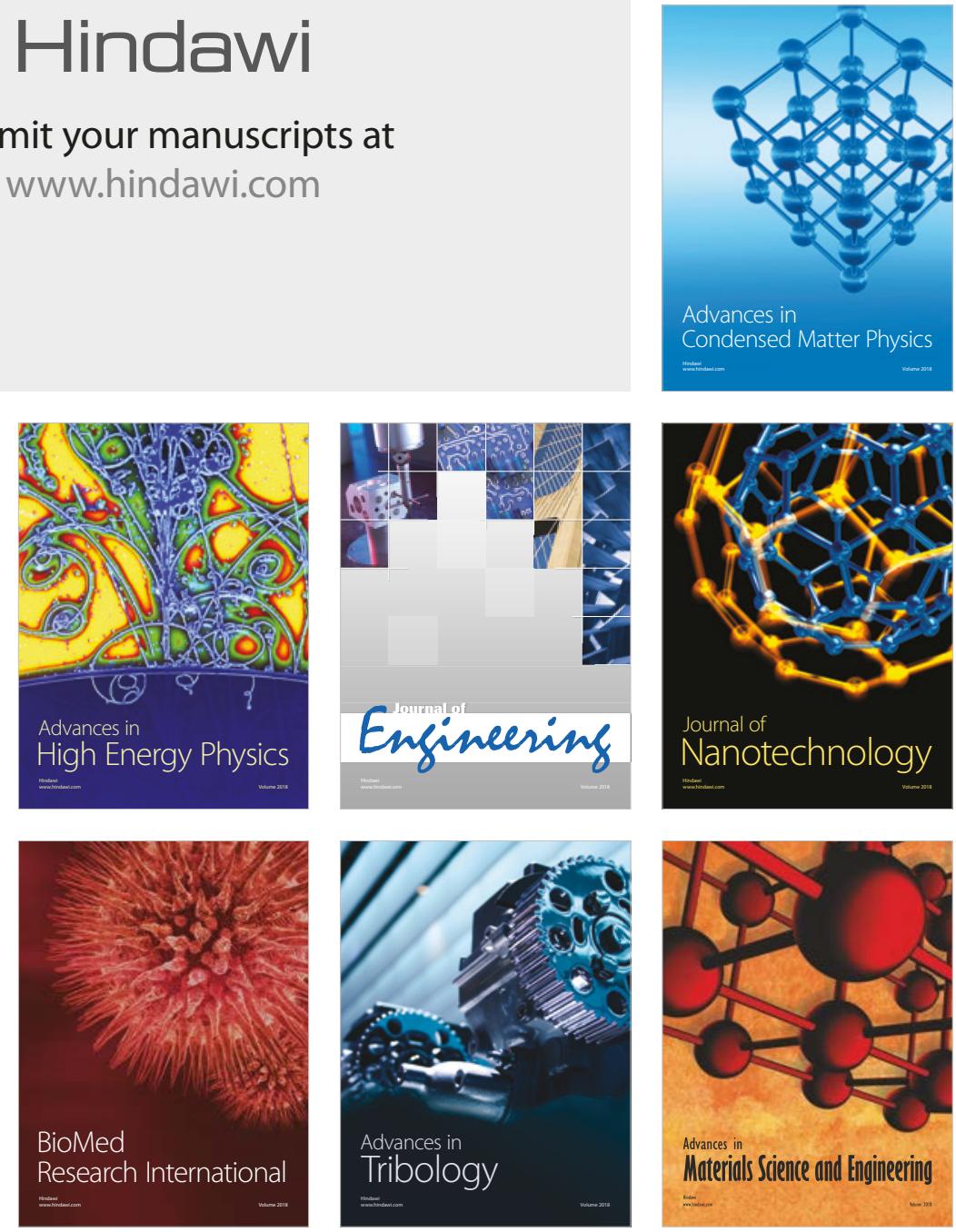